ARTICLE

\title{
An approach to the management of locally advanced breast cancer: Part 1
}

P Govender, MB ChB, FC Rad Onc (SA), MMed, Certification in Medical Oncology European Society for Medical Oncology (ESMO)

Department of Radiotherapy and Oncology, University of KwaZulu-Natal, Durban, South Africa

Corresponding author: P Govender (govenderp10@ukzn.ac.za)

Locally advanced breast cancer (LABC) comprises a heterogeneous group of diseases. It incorporates a subset of stage IIB (T3N0) disease, stage III disease and inflammatory breast cancer. In the developed world, $7 \%$ of breast cancer patients have stage III disease at diagnosis. In developing countries, LABC constitutes about 30 - $60 \%$ of all cases, most probably attributable to a lack of education and poor socioeconomic status. The Surveillance, Epidemiology, and End Results (SEER) Program of the National Cancer Institute, USA demonstrates a median survival of 4.9 years, while the 5 -year relative survival rate for this group of women is $55 \%$ with multimodality treatment, excluding biologics.

S Afr Med J 2014;104(5):384. DOI:10.7196/SAMJ.8245

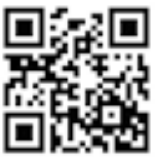

Locally advanced breast cancer (LABC) comprises a heterogenous group of diseases, which incorporates a subset of stage IIB (T3N0) disease, stage III disease and inflammatory breast cancer (IBC) (Fig. 1). In the developed world, based on data from the Surveillance, Epidemiology, and End Results (SEER) Program of the National Cancer Institute, USA, $7 \%$ of breast cancer patients have stage III disease at diagnosis. This is in stark contrast to developing countries, where LABC constitutes 30 - $60 \%$ of all cases, probably because of a lack of education and poor socioeconomic status. SEER data demonstrate a median survival of 4.9 years, while the 5-year relative survival rate for this group of women is $55 \%$ with multimodality treatment, excluding biologics. ${ }^{[1,2]}$

IBC is a rare, distinct subtype of breast cancer associated with rapid disease progression and characterised by erythema and dermal oedema (peau d'orange) of a third or more of the skin area of the breast, with a palpable border to the erythema. ${ }^{[3]}$

\section{Initial patient evaluation} All cases of LABC should ideally be discussed by a multidisciplinary team comprising an oncologist, a breast surgeon, a radiologist, a nurse and a pathologist.

A detailed history of the presenting symptoms, previous breast pathology, risk factors for breast cancer, medical and surgical comorbidities and, in the pre-menopausal female, aspects surrounding family planning and fertility, are important.

This should be followed by a complete clinical examination and blood tests, including a full blood count, a liver function test and alkaline phosphatase levels. Abnormalities in the latter two tests may point towards liver and bone metastases, respectively. In South Africa's HIV-endemic population group, HIV testing is important owing to a higher risk of chemotherapy-induced haematological toxicities in immunocompromised patients. A diagnostic bilateral mammogram (if physically possible) and ultrasound imaging, as well as a chest radiograph to exclude pulmonary metastases, are also part of the mandatory work-up. ${ }^{[3]}$

Core needle breast biopsy is the standard diagnostic procedure as it results in minimal tumour perturbation while offering important diagnostic information, including the identification of tumours that are predominantly or completely located in situ. Additional information gleaned from a core needle biopsy includes the evaluation of the necessary prognostic and predictive tumour biomarkers, e.g. oestrogen receptor/ progesterone receptor (ER/PR), HER2-neu, tumour grade and the Ki67 proliferation marker. This is especially important in the setting of neoadjuvant chemotherapy (NAC) as it may be the only available tumour present if subsequent pathological complete response (pCR) is achieved. ${ }^{[4]}$

While the National Comprehensive Cancer Network (NCCN) guidelines ${ }^{[3]}$ suggest that bone scans and abdominal imaging should be directed by symptoms and other study results, it is standard practice at our institution for patients with LABC to undergo an initial isotope bone scan to exclude bone metastases and an abdominal ultrasound to exclude liver metastases. [18F]-fluorodeoxyglucose (FDG) positron emission tomography/ computed tomography (PET/CT) is primarily used when there are suspicious or equivocal findings on standard imaging studies. An assessment of the left ventricular ejection fraction with an echocardiogram or multigated acquisition (MUGA) scan is mandatory when considering the use of potentially cardiotoxic anthracyline-based chemotherapeutic agents.

\section{Neoadjuvant chemotherapy Rationale}

NAC refers to chemotherapy prior to surgery and is the current standard of care for LABC. It has many potential benefits over immediate surgery and postoperative adjuvant chemotherapy in the management of LABC, which include eradication of occult distant micrometastases and downstaging of a previously unresectable breast malignancy to an operable one. Another

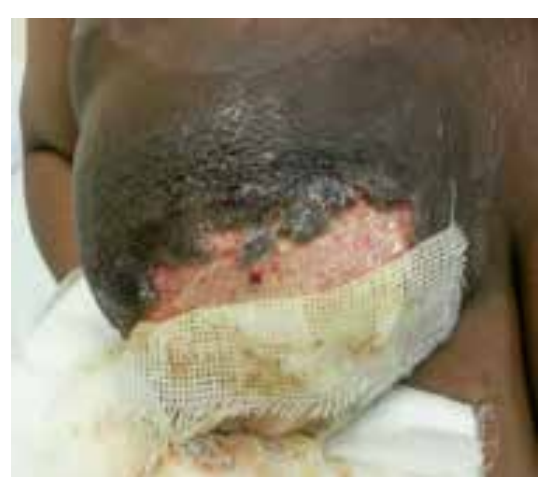

Fig. 1. Locally advanced carcinoma of the right breast. 
advantage of NAC is the opportunity to monitor tumour response to therapy. ${ }^{[5,6]}$

It is noteworthy that, despite the benefits associated with the use of NAC, there is no difference between disease-free and overall survival of patients treated with this mode of chemotherapy compared with adjuvant therapy ${ }^{[6]}$

\section{Regimens}

Historically, regimens based on anthracycline (adriamycin or epirubicin) and cyclophosphamide with or without fluoropyrimidines, e.g. 5-deoxyfluorouridine, formed the backbone of NAC regimens. Secondgeneration trials focused on the addition of taxanes (paclitaxel or docetaxel), either in combination or used sequentially with anthracycline-based regimens. The latter demonstrated improved $\mathrm{pCR}$ rates and avoidance of further postoperative adjuvant chemotherapy. ${ }^{[6]}$

Based on clinical trial data, unless there is clear evidence of disease progression, deviations from the planned therapy in clinical non-responders do not increase pCR or clinical response rate (cRR) or improve survival. Therefore, both responders and non-responders to four cycles of an initial anthracycline-based regimen benefit from crossover to noncross-resistant therapeutic intervention, usually including a taxane. Most of the systemic chemotherapy should be administered before the local treatment. ${ }^{[1]}$

\section{Pathological complete response as a predictor of survival}

Based on current levels of evidence, pCR (ypT0/is, ypN0) has been repeatedly confirmed as the most important prognostic factor and surrogate marker for longer survival in patients receiving NAC. While the ideal result for a patient undergoing NAC is eradication of the malignancy in the breast and the axillary lymph nodes, some residual invasive and/or pre-invasive disease may be identifiable by the pathologist after surgery. ${ }^{[7]}$

The residual cancer burden (RCB) may be used to assess the remaining disease by combining the diameter of the residual primary cancer and the cellularity fraction of the invasive cancer with the diameter of the largest metastasis in the regional lymph nodes in a formula called the RCB Index. By utilising measurements made on routine pathology samples, the RCB Index identifies near pCR and subgroups of resistant cancers and has been validated as a predictor of distant relapse following NAC. ${ }^{[7]}$

\section{Evaluating} chemotherapy response based on molecular subtypes of breast cancer A clinically relevant molecular classification of breast cancer has been introduced, incorporating surrogate markers that would allow for the identification of molecular subtypes using the more familiar immunohistochemical approach. The combined evaluation of ER/PR, HER2-neu and Ki67 immunoreactivity approximates the molecular classification of luminal A, luminal B, HER2-enriched and triplenegative breast cancer. This molecular classification has prognostic value and is also predictive of response to NAC. ${ }^{[8]}$

In patients with a luminal A biological phenotype (ER/PR-positive, HER2-negative, and low Ki67), the conventional chemotherapy regimens have a $6.7 \%$ chance of attaining a pCR. The luminal B-type patients (ER/ PR-positive, HER2-neu-negative, high Ki67 and ER/PR-positive, HER2-neu-positive, any Ki67) have an 11 - 22\% pCR rate, with poorer survival reported in this subset of patients when $\mathrm{PCR}$ is not achieved. The triple-negative (ER/PR-negative, HER2-negative) group of breast cancers are a highly proliferative group of tumours, representing a very responsive group with a $28-32 \%$ probability of a $\mathrm{pCR}$. A survival benefit is demonstrated for patients attaining a pCR. ${ }^{[5]}$

\section{Neoadjuvant hormonal therapy}

Neoadjuvant hormonal therapy (NHT) is an effective therapeutic modality for downsizing primary tumours and rendering them operable, as well as increasing conversion rates from mastectomy to breast conservation surgery in post-menopausal, hormone receptor (HR)-positive breast cancer patients. Furthermore, the favourable toxicity profile of NHT makes it a very suitable treatment option for patients unfit for chemotherapy. ${ }^{[9]}$

The evidence suggests that NHT has comparable response rates to NAC. In postmenopausal women, aromatase inhibitors (AIs) have demonstrated better results than tamoxifen. In the majority of studies, the duration of NHT use prior to surgery was between 3 and 4 months. The available data exploring longer treatment duration, using NHT longer than 3 - 4 months, demonstrated additional clinical responses and further tumour down-sizing. ${ }^{[9]}$

HER2-directed therapies Approximately $20 \%$ of breast cancers overexpress the epidermal growth factor receptor HER2-neu, providing a prognostic and predictive marker for improving pCR rates. ${ }^{[5]}$

In tumours with overexpressed or amplified HER2, adding trastuzumab (Herceptin) to NAC achieves higher pCR rates, mostly in combination with anthracyclines and taxanes. New data demonstrate that combinations of chemotherapy and trastuzumab with a second anti-HER2 agent (lapatinib, pertuzumab) lead to higher pCR rates, which may be translated into a survival benefit. ${ }^{[6]}$

One year of adjuvant trastuzumab remains the standard of care after surgery in patients with HER2-positive tumours. ${ }^{[10]}$

\section{Adjuvant radiotherapy}

Radiotherapy can usually start within 2 - 4 weeks of surgery. Patients receiving chemotherapy can mostly begin radiotherapy 3 weeks after the last cycle of chemotherapy ${ }^{[11]}$

\section{Whole breast radiotherapy}

All patients undergoing breast conservation surgery require adjuvant whole breast radiotherapy (Fig. 2). The target volume is the entire breast using tangential fields treated with $45-50$ Gy in 1.8 - 2 Gy fractions, daily from Monday to Friday. A tumour bed boost with electrons to $60-66 \mathrm{~Gy}$ is recommended. Both computed tomography (CT) scanning and the use of surgical clips have been shown to improve accuracy of localisation of the tumour volume, tumour bed depth and choice of electron energy compared with clinical assessment alone. ${ }^{[12]}$

\section{Post-mastectomy radiotherapy}

Indications for post-mastectomy radiotherapy (PMRT) include patients with T3, T4 tumours and those with $\geq 4$ positive axillary nodes. In patients with 1 - 3 positive axillary nodes, locoregional radiotherapy may be considered for young patients, large T2 - 3 tumours, grade III tumours, ER-negative, lymphovascular invasion or lobular histology. ${ }^{[12]}$

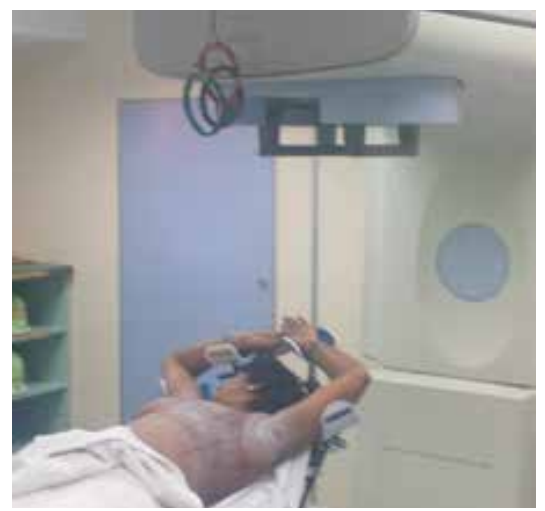

Fig. 2. Left chest wall radiation therapy. 


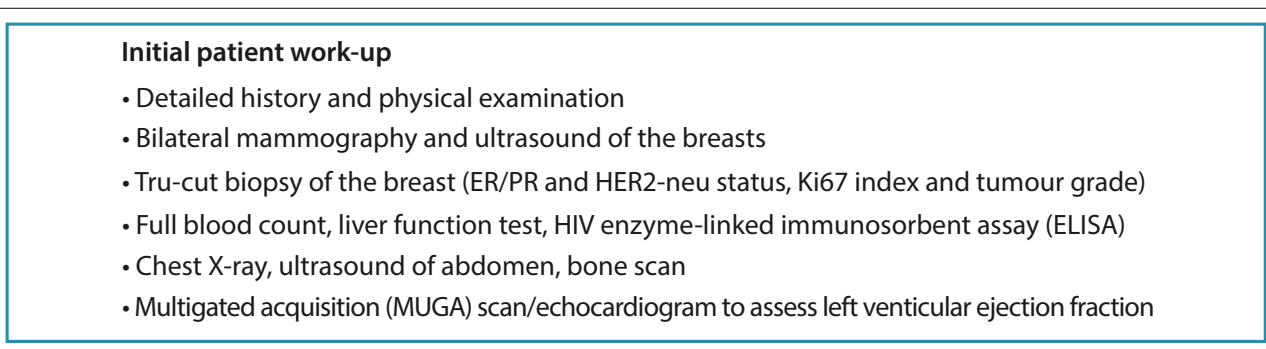

几

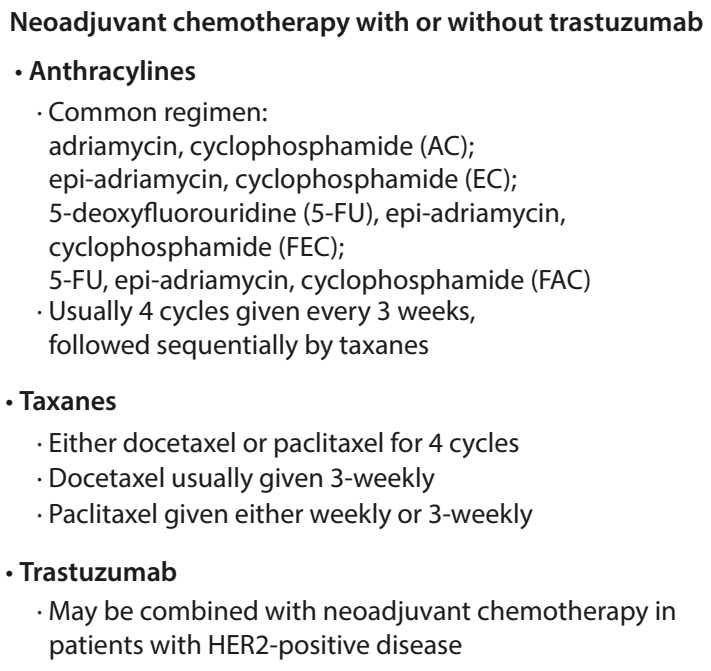

\begin{tabular}{|l} 
Neoadjuvant chemotherapy with or without trastuzumab \\
- Anthracylines \\
. Common regimen: \\
adriamycin, cyclophosphamide (AC); \\
epi-adriamycin, cyclophosphamide (EC); \\
5-deoxyfluorouridine (5-FU), epi-adriamycin, \\
cyclophosphamide (FEC); \\
5-FU, epi-adriamycin, cyclophosphamide (FAC) \\
- Usually 4 cycles given every 3 weeks, \\
followed sequentially by taxanes \\
- Taxanes \\
· Either docetaxel or paclitaxel for 4 cycles \\
- Docetaxel usually given 3-weekly \\
. Paclitaxel given either weekly or 3-weekly \\
- Trastuzumab \\
- May be combined with neoadjuvant chemotherapy in \\
patients with HER2-positive disease
\end{tabular}
Neoadjuvant hormonal therapy
- Usually reserved for HR-positive post-menopausal and HR-positive women who are unfit for chemotheraphy
- Usually administered for 3 - 4 months
- Aromatase inhibitors (Als) preferred over tamoxifen in post-menopausal women

$\sqrt{1}$

Surgery
Wide local excision and axillary lymph node dissection
or
Total mastectomy and axillary lymph node dissection with or without immediate breast reconstruction

ก

Adjuvant radiotherapy
Whole breast radiotherapy
- Recommended for all patients after breast conservation surgery/wide local excision
Post-mastectomy chest wall radiotherapy
- Recommended for tumours $\geq 5 \mathrm{~cm}$ in diameter (T3) and $\geq 4$ axillary nodes involved
- Select high-risk patient population groups with 1 - 3 axillary nodes involved
Regional lymph node irradiation
- Supraclavicular node irradiation
Recommended for patients with $\geq 4$ axillary nodes involved
- Axillary node irradiation
Recommended for patients with an incompletely dissected axilla or extracapsular axillary node extension
- Internal mammary node irradiation
Not routinely recommended unless clinically involved

ᄀ1

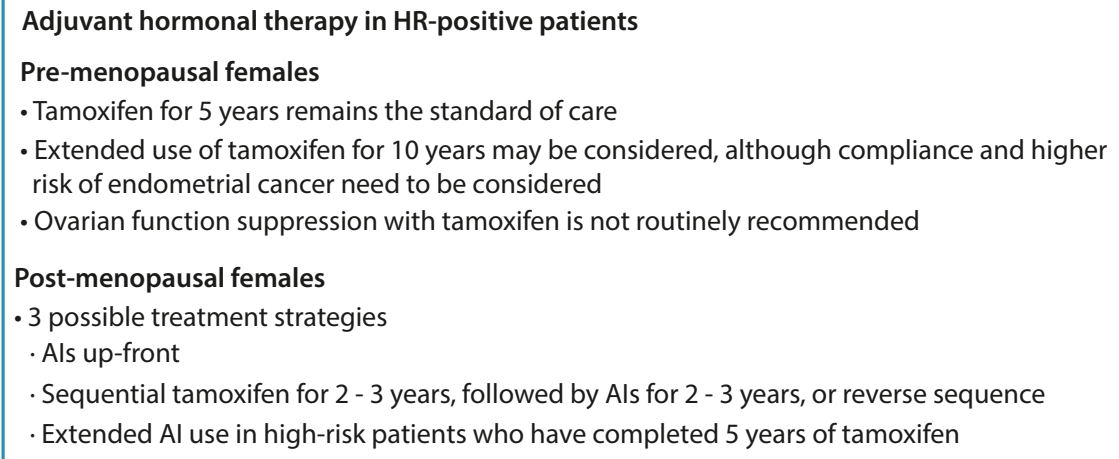

Fig. 3. Proposed treatment algorithm for the management of locally advanced breast cancer. 
Radiotherapy is delivered using opposed tangential fields to encompass the ipsilateral chest wall to a dose of $45-50$ Gy in 1.8 - 2 Gy fractions, daily from Monday to Friday. 3D CT scan planning is recommended to ensure adequate coverage of target, while limiting the dose to the lungs and heart. ${ }^{[13]}$

In a patient who underwent immediate breast reconstruction after mastectomy, conformal or intensity modulated radiotherapy (IMRT) techniques should be used to optimise homogeneity of dose, and bolus irradiation should be avoided if possible to maintain good cosmesis. ${ }^{[12]}$

\section{Regional lymph node irradiation}

The risk of microscopic invasion of the supraclavicular lymph nodes is largely influenced by the number of involved axillary nodes. Supraclavicular lymph node irradiation is indicated when 4 or more axillary nodes are invaded, usually suggesting a $15-20 \%$ risk of supraclavicular lymph node involvement. With conventional fractionation (1.8 - $2 \mathrm{~Gy} /$ fraction) to a total dose of $45-50 \mathrm{~Gy}$, the risk of side-effects remains low. ${ }^{[14]}$

Axillary nodal irradiation is indicated if the patient has had an inadequate axillary dissection, or if there is evidence of extracapsular nodal extension. The lateral border of the supraclavicular field incorporates the axilla. The axillary and supraclavicular lymph node stations are treated using an anterior field with or without a posterior axilla boost field to a total dose of $45-50$ Gy in 1.8 - 2 Gy fractions. ${ }^{[13]}$

The incidence of internal mammary node (IMN) involvement varies between $3 \%$ and $65 \%$, depending on the tumour stage and location of the primary tumour in the breast. However, clinically apparent invasion of these nodes is only $2 \%$. As for the role of IMN radiotherapy, no trial has included sufficient patients or has had sufficient follow-up to definitively evaluate the value of IMN irradiation. If obvious clinical involvement of the IMN chain is present, the IMN can be encompassed by wide tangential fields with cardiac and lung shielding or a separate matched IMN field. ${ }^{[13,14]}$

\section{Adjuvant hormonal therapy Pre-menopausal females}

The standard adjuvant hormonal therapy in pre-menopausal women with ER-positive disease is tamoxifen alone for 5 years, but benefit has also been shown with ovarian function suppression (OFS) using luteinising hormone-releasing hormone (LHRH) agonists, specifically in the absence of chemotherapy. Nonetheless, the use of OFS added to chemotherapy, tamoxifen or a combination of both is not routinely recommended. ${ }^{[10]}$

\section{Post-menopausal women}

The AIs, anastrozole, letrozole and exemestane, have been widely investigated in the adjuvant setting. These drugs may be used as up-front therapy for 5 years, 'switch' strategy of initial tamoxifen for
2 - 3 years, followed by an AI for 2 - 3 years, the reverse sequence, or as an extended treatment after 5 years of tamoxifen, especially in nodepositive patients. Because of the heightened risk of osteoporosis with AI use, guidelines recommend surveillance of bone mineral density during AI treatment, and calcium and vitamin D supplementation, or a bisphosphonate, depending on the result. ${ }^{[10]}$

\section{Extended endocrine therapy}

Due to the risk of recurrence in HR-positive disease after the first decade, clinicians and researchers have been questioning the benefit of extended tamoxifen treatment after 5 years. Three prospective trials (NSABP-B14, aTTom and ATLAS trials) addressed this question, randomising patients after 5 years of tamoxifen treatment to an additional 5 years of treatment or placebo. ${ }^{[10]}$ Except for the NSABP-B14 trial, these studies have demonstrated benefit for extended tamoxifen usage. However, 10 years of tamoxifen is associated with an increased incidence of endometrial cancer (2.3fold with 5 years and 4 -fold with 10 years of tamoxifen). Tamoxifen did, however, show a favourable effect on lipid profile. ${ }^{[10]}$

It is worthwhile noting that compliance when on hormonal treatment is challenging, with reports suggesting that adherence to tamoxifen falls to $50 \%$ during the course of therapy. Non-adherence to anastrozole has been reported in approximately one-third of patients. Of patients taking AIs, the main reason for treatment discontinuation is musculoskeletal toxicities. ${ }^{[10]}$

A proposed treatment algorithm for the management of LABC is given above (Fig. 3).

\section{References}

1. Papademetriou K, Ardavanis A, Kountourakis P. Neoadjuvant therapy for locally advanced breast cancer: Focus on chemotherapy and biological targeted treatments' armamentarium. J Thorac Dis 2010;2(3):160-170

2. Rustogi A, Budrukkar A, Dinshaw K, Jalali R. Management of locally advanced breast cancer: Evolution and current practice. Cancer Res Ther 2005;1(1):21-30. [http://dx.doi.org/10.4103/0973-1482.16086]

and current practice. Cancer Res Ther 2005;1(1):21-30. [http://dx.doi.org/10.4103/0973-1482.16086] National Comprehensive Cancer Network. Breast Cancer (Version 1.2014).
professionals/physician_gls/pdf/breast.pdf (accessed 15 February 2014).

professionals/physician_gls/pdf/breast.pdf (accessed 15 February 2014).
4. Mamounas EP. The impact of neoadjuvant chemotherapy on loco-regional treatment of breast cancer. Curr Breast Cancer Rep 2013;5:106-117. [http://dx.doi.org/10.1007/s12609-013-0106-z] Curr Breast Cancer Rep 2013;5:106-117. [http://dx.doi.org/10.1007/s12609-013-0106-z] 5. Rapoport BL, Demetriou GS, Moodley SD, et al. When and how do I use neoadjuvant chemotherapy for
breast cancer? Curr Treat Options Oncol 2014;15(1):86-98. [http://dx.doi.org/10.1007/s1 1864-013-0266-0] Del Barco S, Ciruelos E, Tusquets I, et al. SEOM clinical guidelines for the systemic treatment of early breast cancer 2013. Clin Transl Oncol 2013;15(12):1011-1017. [http://dx.doi.org/10.1007/ s12094-013-1084-3]

7hompson AM, Moulder-Thompson SL. Neoadjuvant treatment of breast cancer. Ann Oncol 2012;23(10):231-236. [http://dx.doi.org/10.1093/annonc/mds324]

Viale G. The current state of breast cancer classification. Ann Oncol 2012;23(Suppl 10):207-210. [http://dx.doi.org/10.1093/annonc/mds326]

9. Charehbili A, Fontein DB, Kroep JR, et al. Neoadjuvant hormonal therapy for endocrine sensitive breast cancer: A systematic review: Cancer Treat Rev 2014:40(1):86-89. [http//dx doi.org/10.1016/jctrv201306 001] cancer. A A, Ribiero J, Sousa B, Cardoso F. Optimal approach in early breast cancer: Adjuvant and neoadjuv treatment

S, Park C. Fowble B. Breast cancer. In: Hansen EK, Roach MR, eds. Handbook of Evidencebased Radiation Oncology. 2nd ed. New York: Springer, 2010:263-305.

2. Barrett A, Dobbs J, Morris S, Roques T. Practical Radiotherapy Planning. 4th ed. London: Hodder Education, 2009:265-282.

13. Chadha M. Breast Cancer. In: Lu JJ, Brady LW, eds. Decision Making in Radiation Oncology. Berlin: Springer, 2011:207-260. [http://dx.doi.org/10.1007/978-3-642-13832-4_9]

14. Poortmans P. Evidence based radiation oncology: Breast cancer. Radiother Oncol 2007;84(1):84101. [http://dx.doi.org/10.1016/.j.radonc.2007.06.002] 